\title{
An atomic force microscope study of carbon onions and related nanoparticles
}

\author{
GARGI RAINA* and RAHUL SEN \\ Jawaharlal Nehru Centre for Advanced Scientific Research, Jakkur, Bangalore 560064, India \\ (Communicated by Professor CN R Rao) \\ MS received 30 December 1996
}

\begin{abstract}
Carbon onions are found along with carbon nanotubes and other carbon nanoparticles in the cathodic deposit in the arc-vaporization of graphite. Atomic force microscopy has been used to characterize these particles on the basis of their sizes and shapes. Onion-like particles have three-dimensional, near spherical structure and are distinct from two-dimensional graphitic particles. The spherical shape and height to diameter ratios obtained using atomic force microscope, afford a distinction between onion-like structures and other carbon nanoparticles.
\end{abstract}

Keywords. Atomic force microscopy; carbon onions; fullerenes; carbon nanotubes.

\section{Introduction}

During the course of investigations of fullerenes and carbon nanotubes, another novel carbon species involving concentric shells of fullerenes, popularly called onions, was discovered recently by Ugarte $(1992,1994)$. Onions, being hyperfullerenes, should involve concentric cage structures with six- and five-membered rings. Although, onionlike structures have been described from transmission electron microscopy (TEM) (deHeer and Ugarte 1993; Ugarte 1994; Aiyer et al 1995), it can be difficult to distinguish them from small polyhedral graphitic particles. The main distinction will have to come from the volume to surface ratio of these particles. Furthermore, the shape of a graphitic particle can be more two-dimensional compared to onion-like structure, which has to have a three-dimensional or near-spherical structure. Also given the fact that TEM provides the cross-sectional view of an object in a plane perpendicular to the electron beam, one cannot ideally differentiate between a TEM image of a hemispherical or polygonal cap of nanotubes from that of a spherical or polyhedral graphitic particle. Maiti et al (1993) argue that simulated TEM pictures show that polyhedral cages can appear spheroidal when viewed from a particular high symmetry direction, and polyhedral when viewed from a more general direction. Lu and Yang (1994) have shown that spherical multiple-shell fullerenes are likely the most stable structure of large carbon clusters. One can therefore impose certain restrictions of sizes and shapes of the nanoparticles, observed in atomic force microscope (AFM) images, to be identified as onions. A general feature of onions, that has evolved from TEM studies is that they generally consist of around 10-15 concentric shells of variable diameter. This implies that the diameter of such onions would be around $20-30 \mathrm{~nm}$. We, therefore considered it instructive to investigate the shapes and morphologies of various carbon nanoparticles using AFM.

\footnotetext{
* Author for correspondence
} 


\section{Experimental}

Sputtered Au polycrystalline films were deposited using a POLARON sputter coater, on freshly cleaved muscovite. The surface morphology of the evaporated Au films were
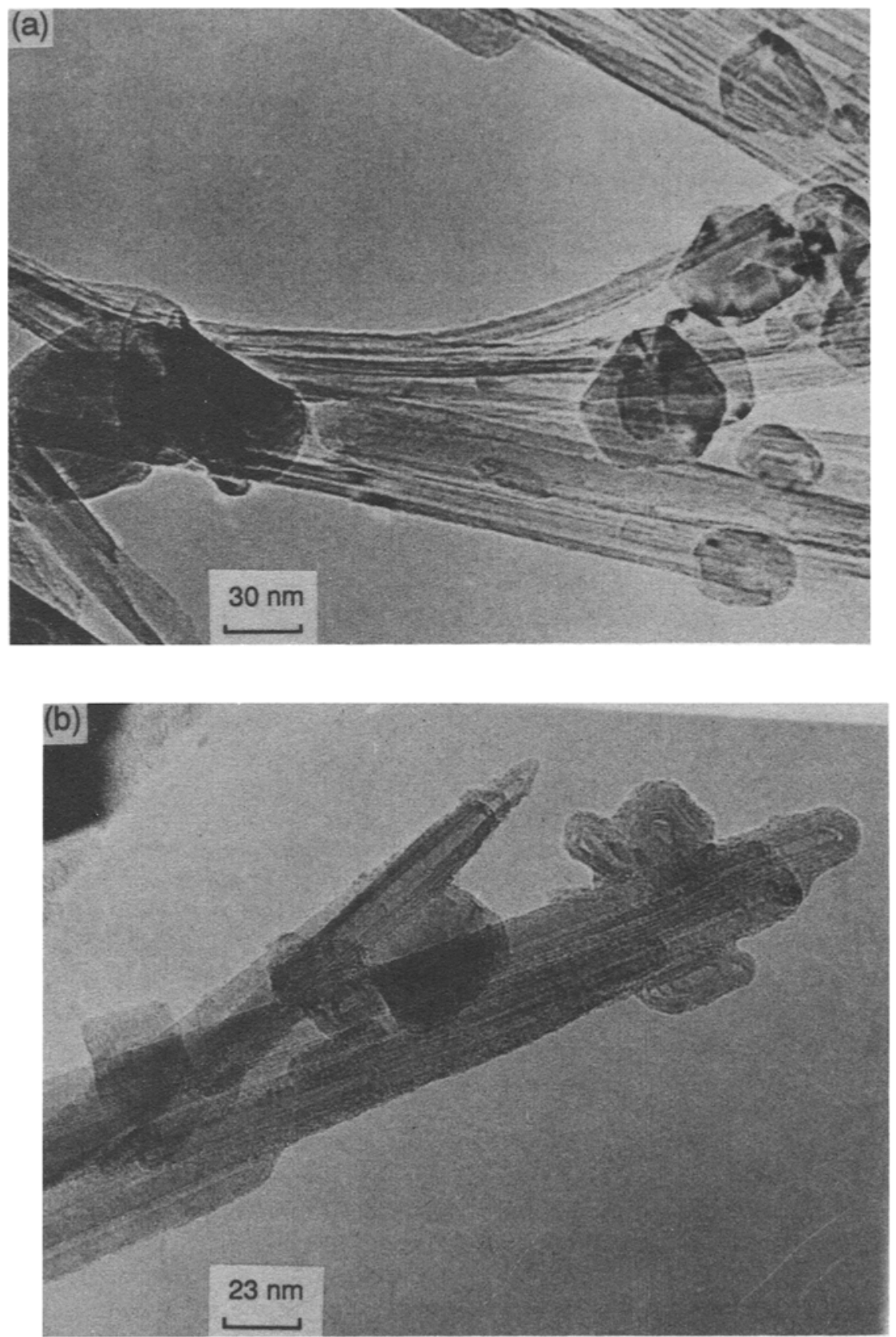

Figure 1. a-b. TEM images of onion-like structures found along with carbon nanotubes in the cathodic deposit obtained in the arc-vaporization of graphite rods. 

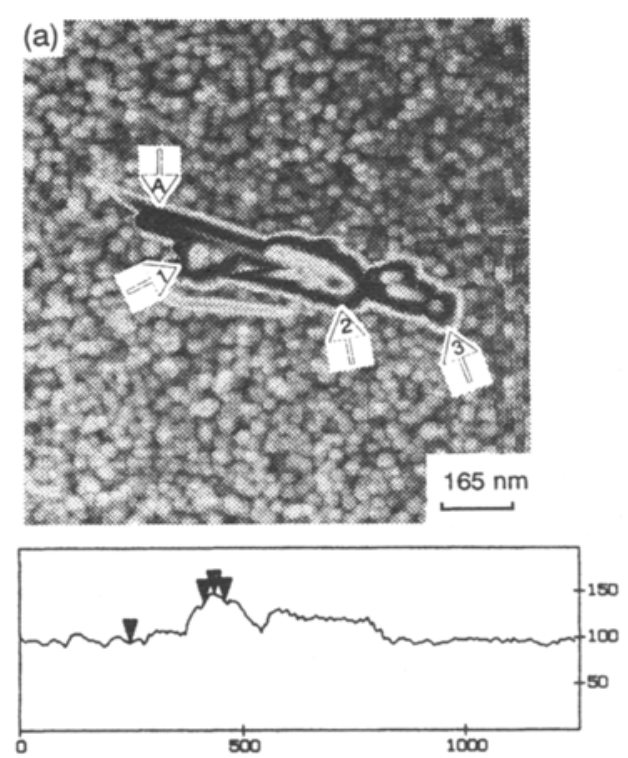
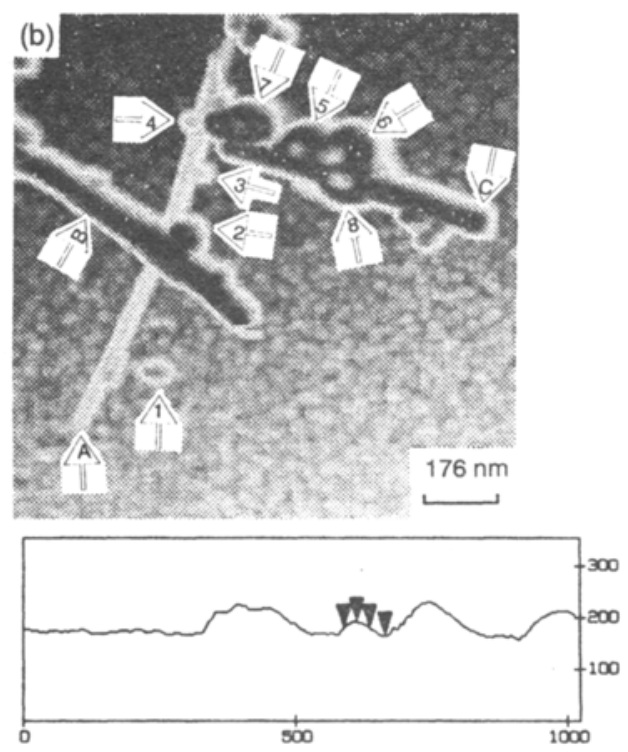

Figure 2. AFM images of carbon nanoparticles present along with carbon nanotubes. a. Shows nanoparticles 1,2 and 3 present along with a nanotube A. Profile of particle 1 is shown below the image, where the diameter is $45 \mathrm{~nm}$ and the height is $53 \mathrm{~nm}$ and b. nanoparticles (1-8) present along with three nanotubes $A, B$ and $C$. Section profile of particle 3 is shown below, where the diameter is $50 \mathrm{~nm}$ and height is $27 \mathrm{~nm}$. The $\mathrm{x}$ - and $\mathrm{y}$-axes scales of the profiles in this and all subsequent figures are in $\mathrm{nm}$.

examined using atomic force microscopy (AFM) and scanning tunneling microscopy (STM). AFM revealed nearly spherical Au grains of $40-50 \mathrm{~nm}$ in diameter and a roughness of about $7 \mathrm{~nm}$. STM revealed smaller particles of about $15-17 \mathrm{~nm}$ in diameter, within these grains.

The core portion of the cathodic stub, obtained in the arc-vaporization of graphite rods, comprises of nanotubes and other graphitic particles. This powder was cleaned by heating in $\mathrm{O}_{2}$ at $480^{\circ} \mathrm{C}$ for $12 \mathrm{~h}$. Drops of the suspension of this powder in $\mathrm{CCl}_{4}$ were let to evaporate on the Au film.

A Nanoscope II, contact-mode AFM was used to characterize the surface morphology of carbon nanotubes and graphitic particles, in air. All the images shown here, were captured in the constant force mode, with an imaging force of the order of $10^{-9}$ Newtons.

\section{Results and discussion}

Onions are generally found along with carbon nanotubes as evidenced in the TEM images of nanotubes as shown in figure 1. In the light of this observation. we first examined the AFM images of carbon nanoparticles present along with carbon nanotubes. In figure 2, we show typical AFM contour plots of onion-like structures accompanying the nanotubes. For example, in figure $2 \mathrm{a}$, we identify three particles designated 1, 2 and 3 which have diameters in the range of $40-70 \mathrm{~nm}$. The heights of 
Table 1. Dimensions of nanoparticles in figure 2.

\begin{tabular}{lcccll}
\hline Figure & Particle \# & $\begin{array}{c}\text { x-y } \\
\text { Dimension } \\
\text { ('D' nm) }\end{array}$ & $\begin{array}{c}\text { Height } \\
\text { ('H' nm) }\end{array}$ & H/D & Description \\
\hline 2 (a) & 1 & 45 & 53 & $1 \cdot 2$ & Spherical \\
& 2 & $50 \times 36$ & 59 & $1 \cdot 2$ & Polyhedral \\
& 3 & $69 \times 36$ & 44 & 0.6 & Polyhedral \\
2 (b) & 1 & $87 \times 50$ & 32 & 0.4 & Polyhedral \\
& 2 & 52 & 55 & $1 \cdot 1$ & Spherical \\
& 3 & 54 & 27 & 0.5 & Spherical \\
& 4 & 59 & 35 & 0.6 & Spherical \\
& 5 & 69 & 33 & 0.5 & Spherical \\
& 6 & $97 \times 87$ & 29 & 0.3 & Polyhedral \\
& 7 & $118 \times 72$ & 46 & 0.4 & Polyhedral \\
& 8 & $66 \times 50$ & 51 & 0.8 & Polyhedral \\
\hline
\end{tabular}
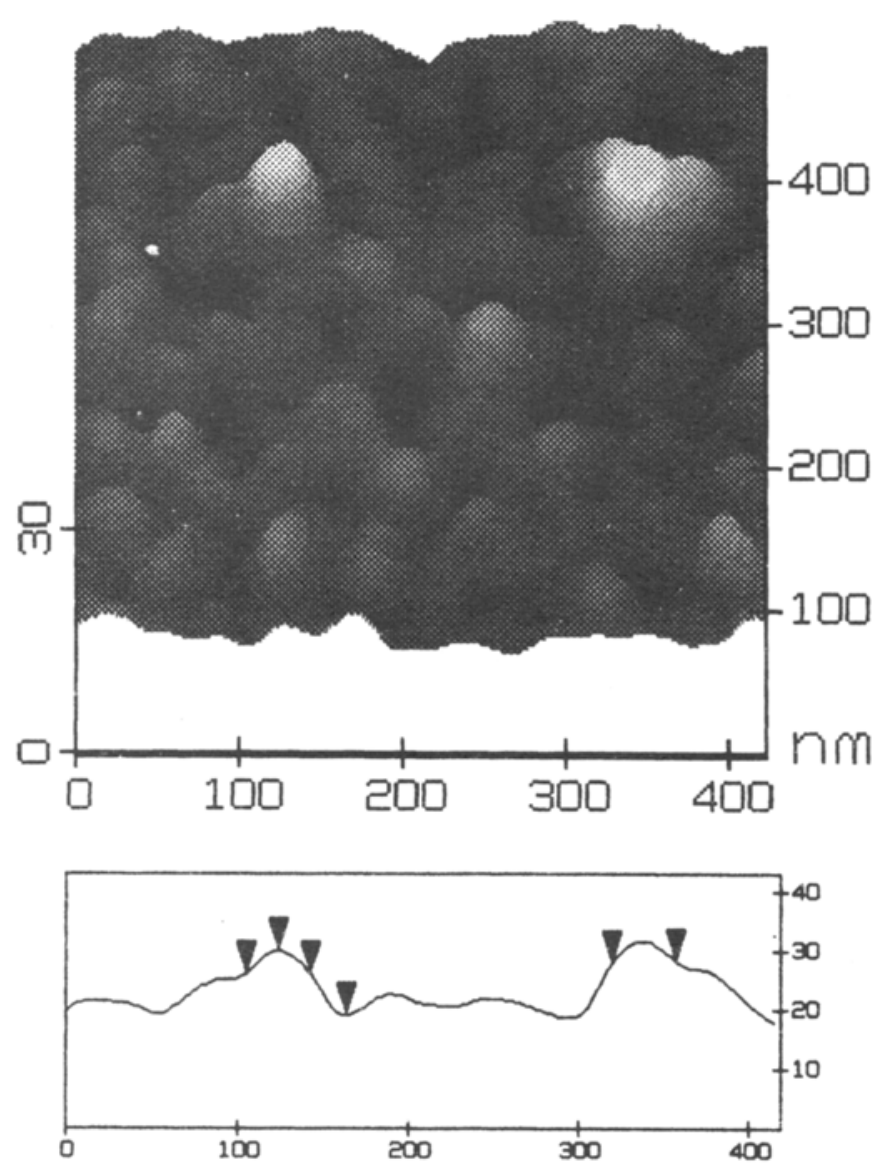

Figure 3. Surface plot of two isolated onion-like particles, with the section profile across them shown below, where the diameters are $37 \mathrm{~nm}$ each and the height is $11 \mathrm{~nm}$. 
these particles vary between $40-60 \mathrm{~nm}$. Of these, particle 1 is closest to being spherical and may be considered to be onion-like. The line profile across particle 1 shown below the image, supports this conclusion. In figure $2 b$, we see the presence of $a$ large number of onion-like particles with three nanotubes. Particles marked 1-6, all have characteristic diameters in the range of 50-90 nm and heights in the range of $30-50 \mathrm{~nm}$. We show a line profile of particle 3 in the figure. Particles 2-5 are close to being spherical. Compared to these, particles 7 and 8 are considerably larger, with $\mathrm{x}-\mathrm{y}$ dimensions of around $120 \mathrm{~nm} \times 70 \mathrm{~nm}$ and with heights in the $30-40 \mathrm{~nm}$ range, suggesting that they are unlikely to be onions. In table 1 , we list the dimensions of the various particles.

In addition to onion-like carbon nanoparticles found along with nanotubes, we have imaged a number of isolated carbon nanostructures, some of which are fairly spherical. Their sizes vary widely. We briefly describe the morphology of these carbon nanoparticles. In figure 3, we show the surface plot of an AFM image showing two isolated particles whose diameters are $37 \mathrm{~nm}$ each and heights of $11 \mathrm{~nm}$ and $12 \mathrm{~nm}$, which are not far from onion-like structures. We have imaged particles still larger than those in figure 3 , some of which are spherical. We cannot, however, be certain that they are onion-like since the height to diameter $(\mathrm{H} / \mathrm{D})$ ratio is much smaller than one would expect for onions.

In figure $4 a$, we show an image along with the line profile of a particle whose diameter is $290 \mathrm{~nm}$, which appears to be spherical but the height is only $40 \mathrm{~nm}$. In figure $4 \mathrm{~b}$, we see a large spherical arrangement consisting of a large particle along with small spherical particles whose diameters are around $70 \mathrm{~nm}$ and height $35 \mathrm{~nm}$, which are quite onion-like. This may suggest occurrence of packing of the onion-like particles. Ru et al (1996) have demonstrated that carbon onions formed in TEM also show some aggregation phenomenon. These images are compared with those of polyhedral particles which do not show any apparent spherical shape. In figure 4c, we show an AFM contour plot of two polyhedral particles. The line profile across these two particles along one direction gives the diameters to be $230 \mathrm{~nm}$ and $250 \mathrm{~nm}$, with heights of $101 \mathrm{~nm}$ and $124 \mathrm{~nm}$, respectively. Table 2 lists the dimensions of the various particles in figure 4 .

\section{Conclusions}

We have studied the shapes and sizes of different carbon nanoparticles obtained in the arc-vaporization of graphite rods, using AFM. Particles for which the H/D ratio is greater than 0.3 are considered to be three-dimensional structures and those with less than 0.3 are considered to be two-dimensional or planar structures. Particles with distinct $x-y$ dimensions are considered to be polyhedral vis- $a$-vis those with similar $\mathrm{x}-\mathrm{y}$ dimensions are considered to be spherical. We find three types of carbon nanoparticles.

Type 1: This type of nanoparticles occur along with or attached to nanotubes and have typical sizes in the range of $40-70 \mathrm{~nm}$. Most of these particles are spherical or nearspherical in shape with the height to diameter ratios of greater than $0 \cdot 3$. Such particles can be identified as carbon onions because of their reasonably small size and three-dimensional shape.

Type 2: These are isolated particles with diameters in the range of $30-40 \mathrm{~nm}$ and heights in the range of $10-12 \mathrm{~nm}$. The height to diameter ratio is around 0.3 and hence, they are not far from onion-like structures. 

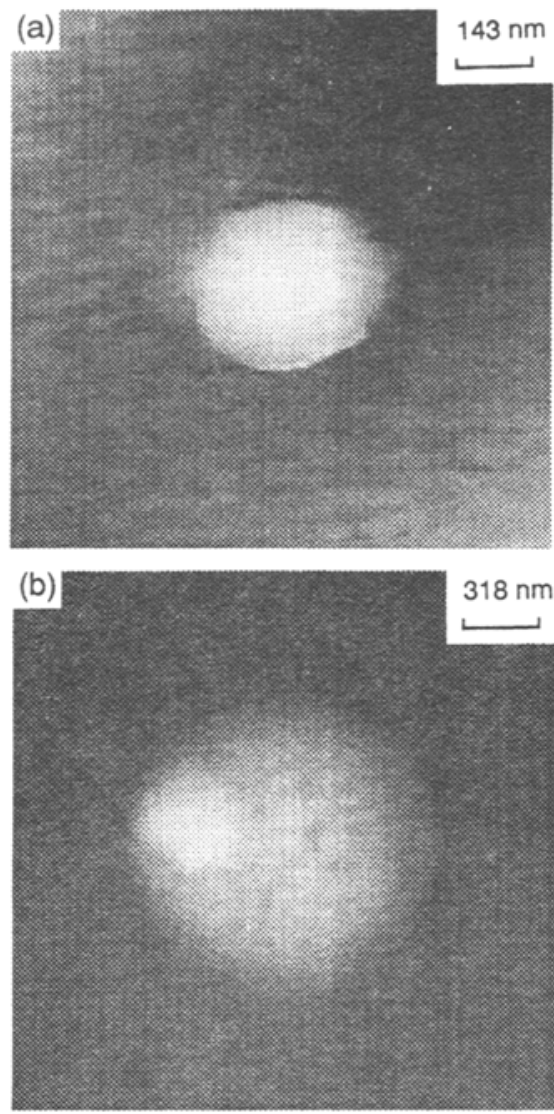

(c)

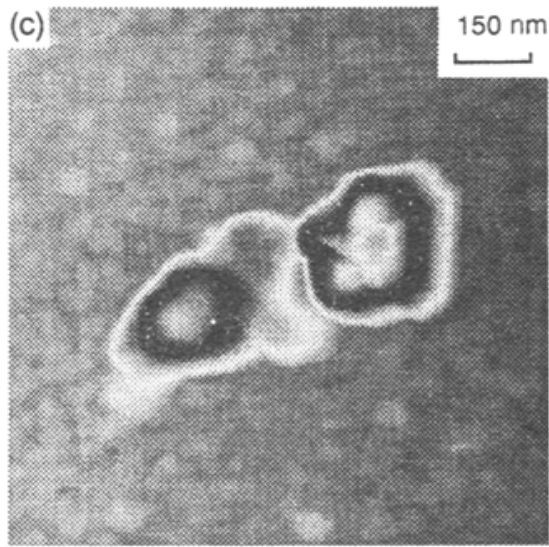

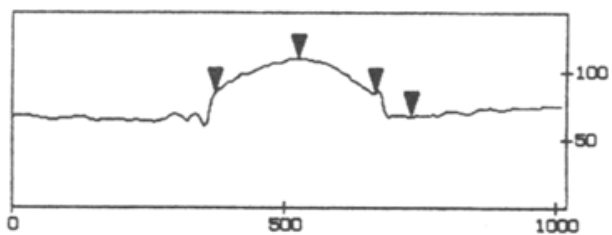
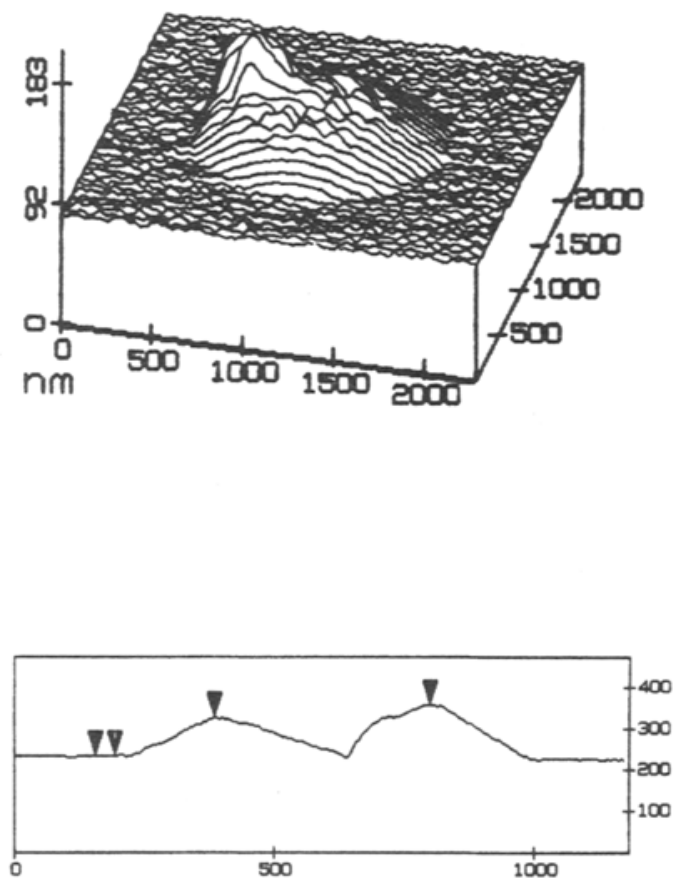

Figure 4. AFM images of large carbon nanostructures. a. Top-view of a large spherical structure with section profile shown alongside, where the diameter is $294 \mathrm{~nm}$ and height is $44 \mathrm{~nm}$, b. image showing a large and several small particles arranged in a spherical shape. A lineplot is shown on the side and c. contour plot of two non-spherical particles with a line profile across them, giving heights of $101 \mathrm{~nm}$ and $124 \mathrm{~nm}$, respectively. 
Table 2. Dimensions of nanoparticles shown in figure 4.

\begin{tabular}{lcccll}
\hline Figure & Particle \# & $\begin{array}{c}\text { D-y } \\
\text { Dimension } \\
\text { ('D' nm) }\end{array}$ & $\begin{array}{c}\text { Height } \\
\text { ('H' nm) }\end{array}$ & H/D & Description \\
\hline 4(a) & 1 & 290 & 40 & $0 \cdot 1$ & Planar \\
4(b) & 1 & 926 & 53 & $0 \cdot 1$ & Planar \\
& 2 & 72 & 31 & $0 \cdot 4$ & Spherical \\
4(c) & 1 & $251 \times 171$ & 101 & $0 \cdot 4$ & Polyhedral \\
& 2 & $254 \times 232$ & 124 & $0 \cdot 5$ & Polyhedral \\
\hline
\end{tabular}

Type 3: These are very large particles with diameters typically around $250 \mathrm{~nm}$ and their shapes are either spherical or polyhedral. These large particles may be an agglomeration of smaller spherical or polyhedral particles, as revealed sometimes in the AFM images. We observed a very large spherical particle with a diameter of $\sim 920 \mathrm{~nm}$ which is an agglomerate of small onion-like particles.

\section{Acknowledgement}

The authors thank Prof. CNR Rao for suggesting the problem and for the guidance.

\section{References}

Aiyer H N, Seshadri R, Raina G, Sen R and Rao C N R 1995 Full. Sci.\& Tech. 3765 deHeer W A and Ugarte D 1993 Chem. Phys. Lett. 207480

Lu J P and Yang W 1994 Phys. Rev. B49 11421

Maiti A, Brabec C J and Bernholc J 1993 Phys. Rev. Lett. 703023

Ru Q, Okamoto M, Kondo Y and Takayanaki K 1996 Chem. Phys. Lett. 259425

Ugarte D 1992 Nature 359707

Ugarte D 1994 MRS Bulletin XIX 39 